(c) 2012 IEEE. Personal use of this material is permitted. Permission from IEEE must be obtained for all other uses, in any current or future media, including reprinting/republishing this material for advertising or promotional purposes, creating new collective works, for resale or redistribution to servers or lists, or reuse of any copyrighted component of this work in other works. 


\section{Candidate Wire Spiral Antennas for the SKA Radio Telescope}

\author{
Aziz Jiwani, Shantanu Padhi, Mark Waterson and \\ Peter J Hall \\ ICRAR/Curtin Institute of Radio Astronomy (CIRA) \\ Curtin University \\ Perth, Australia \\ aziz.jiwani@icrar.org
}

\author{
Jan Geralt bij de Vaate \\ ASTRON Dwingeloo, The \\ Netherlands vaate@astron.nl
}

\begin{abstract}
The SKA is a next generation radio telescope employing phased aperture arrays at its lowest operating band (SKA-low) of $70-450 \mathrm{MHz}$. SKA-low is a dual-polarized array consisting of antenna elements with stable impedance and low cross-polarization. We select the well-known broadband conical spiral antenna and its dual-polarized derivative to be evaluated as candidate elements for SKA-low. Previously, we evaluated the sheet version of the conical spiral and found that it maintained well its broadband characteristics. Looking towards ease of manufacturing, we now evaluate wire versions of the designs. We find that the single-polarized wire version has stable impedance and low cross-polarization from 150 - $450 \mathrm{MHz}$. However, at low frequencies the cross polarization increases. The dual-polarized version maintains its characteristics over a small band but with higher $(>-20 \mathrm{~dB})$ cross polarizations. Measured results confirm the simulated antenna behavior.
\end{abstract}

\section{Keywords-Antennas; SKA; Radio Astronomy}

\section{INTRODUCTION}

The Square Kilometre Array (SKA) is a next generation radio telescope [1]. Operating from $70 \mathrm{MHz}$ to $>10 \mathrm{GHz}$, the SKA will employ various antenna technologies at different bands [2]. The lowest band (SKA-low), operating at $70-450$ $\mathrm{MHz}$, will consist of groups of phased array antennas, called stations. These stations contain thousands of antennas, can be hundreds of kilometers apart, and will generate steerable beams which are correlated to produce high dynamic range pictures of the sky at radio wavelengths.

The stations are required to sense orthogonal polarizations with raw polarization purity (or cross-polarization) of better than -20 dB [3]. The SKA-low uses active antennas (antennas with LNAs attached to their outputs) so antenna elements need to match the nominal LNA impedance over the required band [3].

Conical log spirals [4] are well known broadband antenna which have reported stable impedance and low crosspolarization [5]. Hence, the conical spiral antenna is selected to be evaluated as a SKA-low candidate element. We expect the wire version of the spiral to be manufacturable in the high

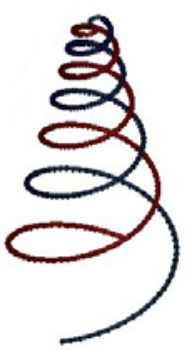

(a)

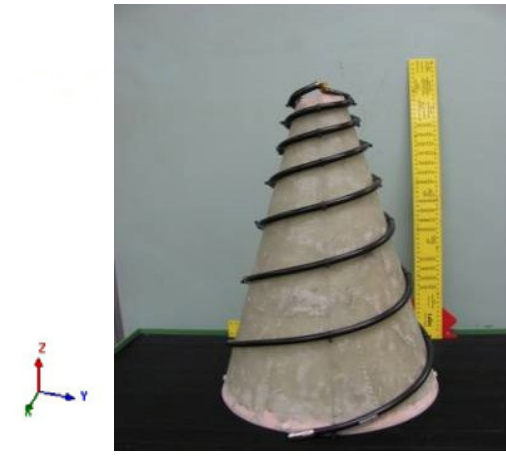

(b)
Figure. 1. Wire conical log spiral antenna (a): model and (b): prototype

volume needed for an SKA station consisting of thousands of antennas. Wire spirals have been reported to retain the characteristics of the sheet spiral [4], motivating our evaluation.

As SKA-low stations require dual polarization, one may choose to compose a station of interspersed opposite-hand spirals. However, this would require twice the number of elements, resulting in higher costs and larger station size. If opposite-wound spirals could be integrated on one former, the resulting station would be simplified. We thus evaluated a dual-polarized modification of the simple spiral.

The current investigation is conducted for wire spirals operating in free space. As the SKA-low station will be placed over either over a metallic ground plane or soil, the next stage of the evaluation will be to investigate the wire antenna performance over these media.

This paper reports the results of the investigation of the single and dual-polarized wire spiral. Section II presents the basic design of the spiral. Results from the simulation and measurement of prototypes are presented in section III and IV respectively. The conclusions from the investigation are reported in section $\mathrm{V}$. 


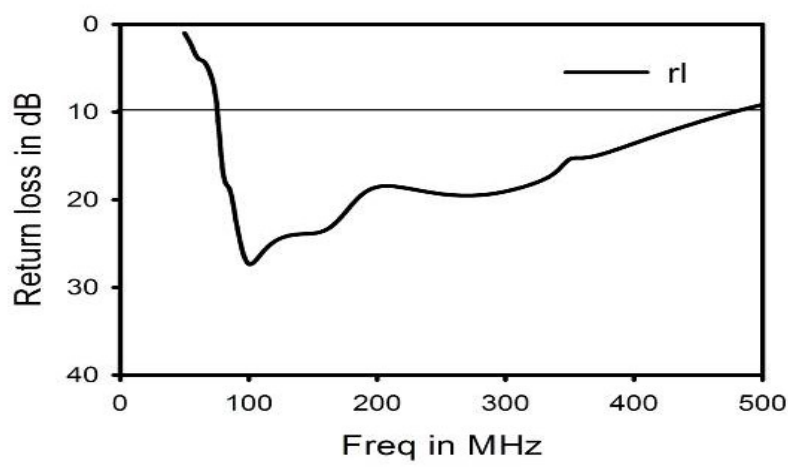

Figure. 2. Calculated return loss of the single polarized wire spiral

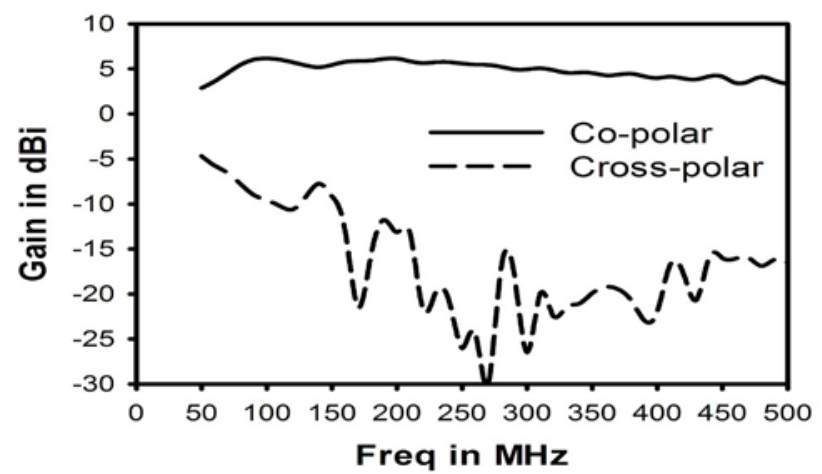

Figure. 3. Calculated co- and cross-polar component of gain of the single polarized wire spiral.

\section{DESIGN OF THE CONICAL LOG SPIRAL}

The log spiral antenna belongs to the frequency independent class of antennas [7]. The design and geometry of the spiral is dependent on three parameters. They are: wrap angle $\alpha$, half cone angle $\theta_{0}$ and strip width (or arm width) $\delta$. The spiral arms are generated by the equation:

$$
\rho_{\mathrm{N}}=\rho_{0} \exp \left(-\sin \theta_{0} / \tan \alpha\right) \Delta \Phi
$$

In this equation, $\rho_{0}$ is the radius vector from the origin to the truncation of the spiral at the apex region, $\rho_{\mathrm{N}}$ is the radius vector to an edge of the arms at a given angle $\Phi$. The other edge of the arm is generated by adding $\delta$ (which is in degrees) to $\Phi$. This enables the spiral arm width to change as a function of frequency [4]. The second arm is generated by rotating the first arm by 180 degrees, or adding $\pi$ radians to the equation describing the edges of the first arms [4].

Replacing the arms of the spiral with constant diameter wires forms the wire conical log spiral. The dual-polarized version of this design has two wire conical log spirals with opposite polarization (or winding sense), but the same spiral parameters. One of the spirals is placed over the other spiral, with a small air gap, to form the dual-polarized antenna.
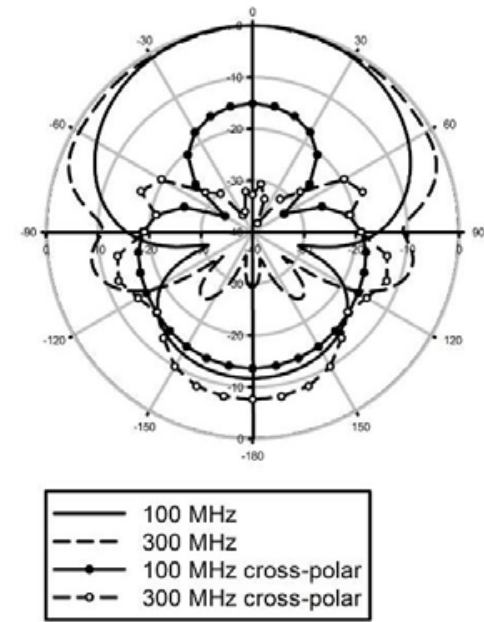

Figure. 4. Calculated normalized (to the co-polar component) radiation pattern of the single polarized wire spiral at phi $=0$.

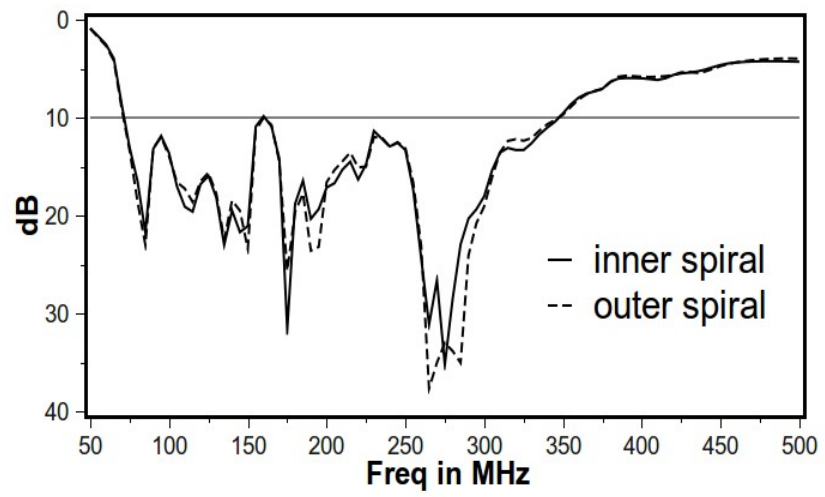

Figure. 5. Calculated return loss of the two polarizations of the wire conical log spiral antenna

\section{SiMULATION RESUlTS}

\section{A. Single-polarized wire conical log spiral}

Larger numbers of turns improves the spiral radiation characteristics and lowers its cross-polarization. The number of turns is directly proportional to wrap angle and inversely proportional to cone angle. Small cone angles result in dimensionally tall (and difficult to manufacture) antennas while higher wrap angles reduce the spacing between the arms (at apex), restricting the allowable size of the wire diameter. The input impedance of the wire spiral is inversely proportional to the wire diameter. For wire diameters $<10 \mathrm{~mm}$, the input impedance is $>300$ ohms.

Thus, from practical considerations, a design with 10.29 mm copper wires (diameter of LMR-400 coaxial cable) having a wrap angle of $75^{\circ}$ and cone angle of $15^{\circ}$ (see Fig. 1a) is simulated. This antenna has an apex diameter of $167 \mathrm{~mm}$, base diameter of $1285 \mathrm{~mm}$ and is $2100 \mathrm{~mm}$ in height. The prototype single-polarization antenna is right-hand circularly polarized (RHCP). 


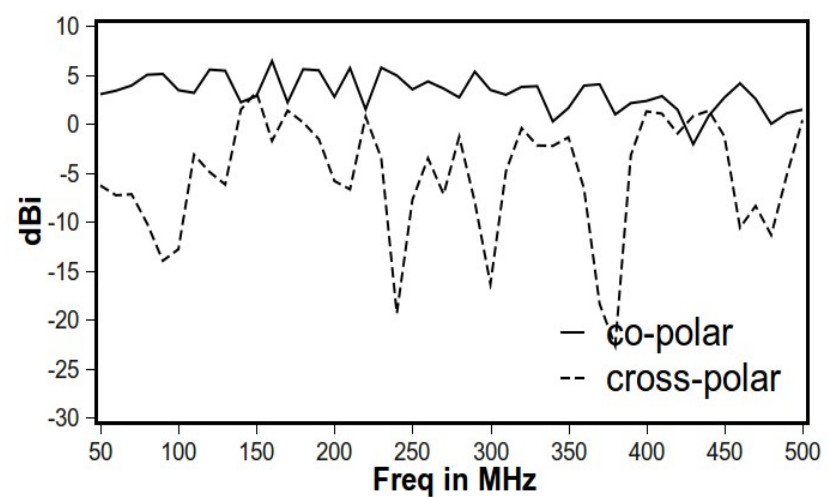

(a)

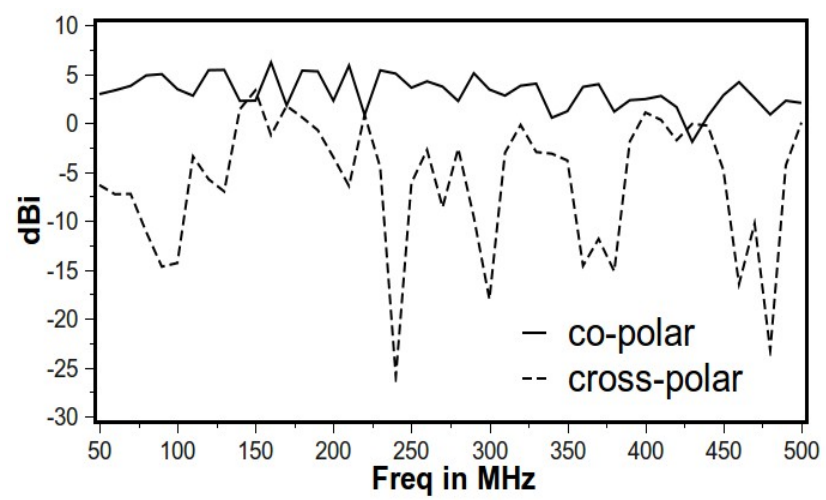

(b)

Figure. 6. Calculated co- and cross-polar component of gain for (a) inner polarization and (b) outer polarization of the dual spiral.

The return loss of the modeled spiral, for reference impedance of 300 ohms, is $>10 \mathrm{~dB}$ over the operating band (as shown in Fig. 2). The co-polar gain of the spiral has undulations but is $>4 \mathrm{dBi}$ over the operating band (as shown in Fig. 3).

The cross-polar level is <-20 $\mathrm{dB}$ above $150 \mathrm{MHz}$ though the value increases to $-7 \mathrm{~dB}$ at $70 \mathrm{MHz}$. The simulated radiation pattern of the single-polarized wire conical log spiral is plotted in Fig. 4. It presents low backlobes $(<-10 \mathrm{~dB})$ and a smooth main lobe.

As a comparison, an identical sheet spiral (not shown) has co-polar gain of $>6 \mathrm{dBi}$ across the operating band with crosspolar levels of $<-20 \mathrm{~dB}$ from $100 \mathrm{MHz}$ onwards. Thus, the copolar gain of the antenna reduces by $2 \mathrm{dBi}$ over the band and the polarization purity is $>-20 \mathrm{~dB}$ over the $100-150 \mathrm{MHz}$ band when we switch from sheet to wire spirals.

The wire spiral meets or exceeds the SKA requirements over the $150-450 \mathrm{MHz}$ band. Increasing the spiral footprint will improve the currently-deficient low frequency characteristic of the wire spiral, making it consistent across the band.

\section{B. Dual-polarized wire conical log spiral}

The dual-polarized antenna is formed by adding another set of spiral arms, with same spiral parameters, to the single-
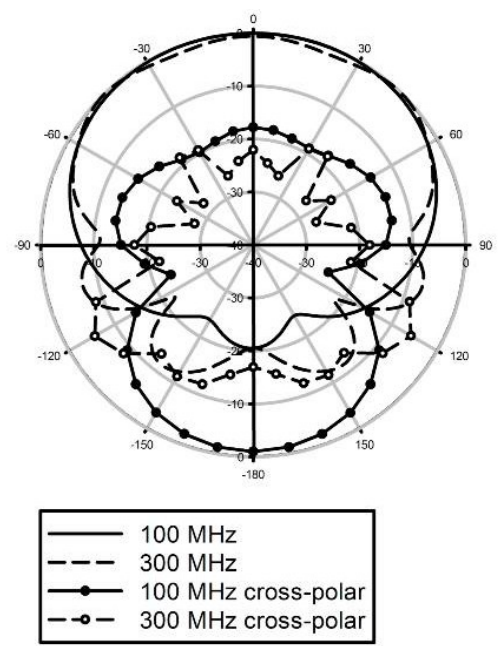

(a)
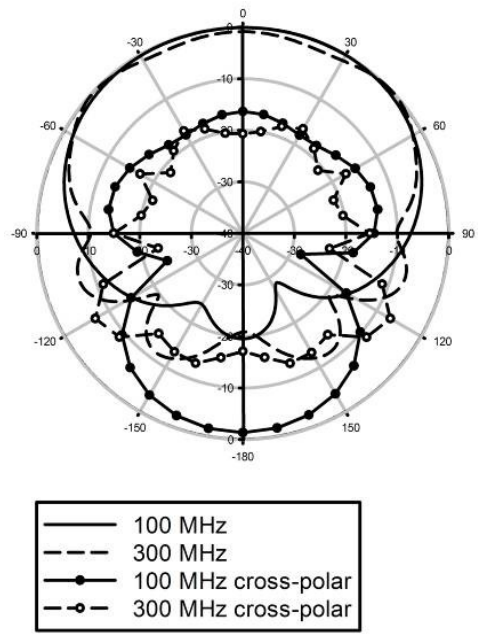

(b)

Figure. 7. Calculated normalized (to the co-polar component) radiation pattern of the (a) inner polarization and (b) outer polarization of the dual polarized

polarized antenna. The second spiral is wound in opposite sense to the first spiral. Dimensionally the apex and base diameter of the second spiral is larger than the first spiral by $20 \mathrm{~mm}$, though the height remains the same.

The dual-polarized wire spiral is optimized for the SKAlow frequency range of $70-450 \mathrm{MHz}$. LMR 400 coaxial cable models are used as spiral arms. The inner spiral is righthand circularly polarized (RHCP) and the outer spiral is a lefthand (LHCP) antenna.

The simulated return loss of the wire conical spiral is $>10 \mathrm{~dB}$ for $70-350 \mathrm{MHz}$ (see Fig. 5) band for an input impedance of 300 ohms. The gain of the dual-polarized spiral is not smooth, though, and contains $\sim 3 \mathrm{~dB}$ ripples (see Fig. 6). The cross-polarization levels are not consistent, though there are ranges of frequencies where the polarization purity is better than $-20 \mathrm{~dB}$. 
The simulated radiation patterns of the two polarizations of the spiral, at 100 and $300 \mathrm{MHz}$, are plotted in Fig. 7 for the inner and outer antennas.

As seen from Fig. 7, the main lobes of the inner and outer spirals at the two frequencies are nearly the same. Similarly, the back lobes are $<-10 \mathrm{~dB}$ and the cross-polarizations are $<-$ $15 \mathrm{~dB}$ at boresight.

Simulated results suggest that this spiral can operate as a dual circularly polarized antenna. However, the bandwidth of the antenna with respect to the return loss, gain and cross polar levels is smaller than the required SKA-low band. Thus, the dual-polarized wire spiral antenna design is not suitable for SKA-low. Interestingly though, the spiral does operate over a wide (3:1) bandwidth with somewhat reduced polarization purity. More work is underway to understand better the dual spiral design and its limitations.

\section{MEASURED RESULTS}

A prototype of the single-polarized wire spiral is measured to confirm the results of the simulation. The prototype is a 3:1 frequency scaled version of SKA-low, operating from 210 $1350 \mathrm{MHz}$. The former for the prototype is constructed from fiberglass resin and LMR-400 coaxial cables are used as spiral arms. The prototype has an apex diameter of $58 \mathrm{~mm}$, base diameter of $321 \mathrm{~mm}$ and is $492 \mathrm{~mm}$ in height.

This prototype is measured in an anechoic chamber and at an outdoor measurement facility. Reference [8] describes the outdoor measurement procedure employed for these prototypes. The measured radiation pattern at two frequencies (500 MHz and $750 \mathrm{MHz}$ ) for the single-polarized prototype is given in Fig. 8. The presented results are from the outdoor measurement procedure. The results of the simulation are also plotted on the same graphs for comparison.

The difference in the measured and simulated data is $<3 \mathrm{~dB}$ over the main lobe. The back lobe is $<-10 \mathrm{~dB}$ for both frequencies, though there is some difference in the measured and simulated results, perhaps because of simplified feed and measurement arrangements. Nevertheless, it can be inferred that the simulations reasonably predict the features of the wire spiral.

\section{CONCLUSION}

Simulations and measured results confirm reported results indicating wideband operation of the single-polarized wire spiral antenna The dual-polarized wire spiral has $>-10 \mathrm{~dB}$ return loss over a wide (though smaller than required) bandwidth; the radiation characteristics are not smooth and contain ripples in gain and low polarization purity. The general behaviour of the dual-polarized spiral makes it unattractive, in our view, for SKA-low.

\section{ACKNOWLEDGMENT}

The authors would like to thank Jonathan Tickner and John Flexman for their support in construction and measurement of the prototype. Franz Schlagenhaufer provided support for anechoic chamber measurements and supplied the sources. Adrian Sutinjo injected much valuable advice and many suggestions.
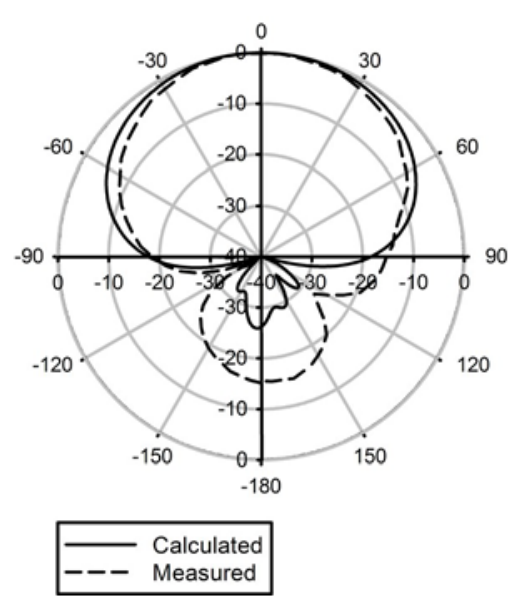

(a)
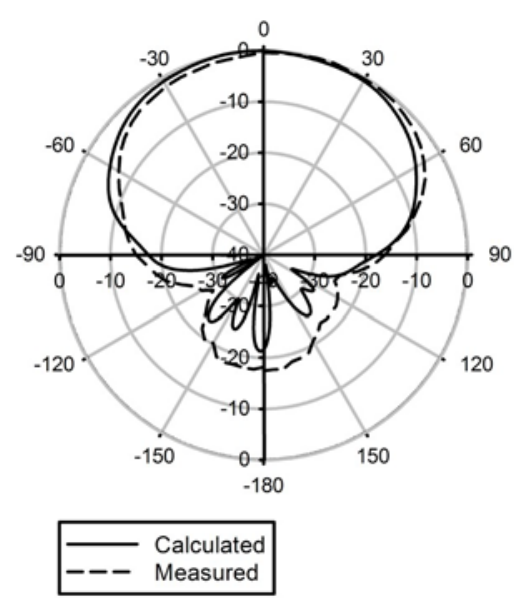

(b)

Figure. 8. Measured and calculated radiation pattern of the single polarized wire spiral for (a) $500 \mathrm{MHz}$ and (b) $700 \mathrm{MHz}$ at phi $=0$.

\section{REFERENCES}

[1] Dewdney, P.E.; et al , "The Square Kilometre Array," Proc IEEE, vol.97, no.8, pp.1482-1496, Aug. 2009.

[2] Schilizzi, R.; et al, "Preliminary specifications for the Square Kilometre Array,” SKA Technical Memo Series, vol. 2, no. 1, p. 4, 2007.

[3] de Vaate, J.G.B.; et al , "Low frequency aperture array developments for phase 1 SKA," General Assembly and Scientific Symposium, 2011 XXXth URSI, vol., no., pp.1-4, 13-20 Aug. 2011

[4] Dyson, J.;, "The unidirectional equiangular spiral antenna," Antennas and Prop, IRE Transactions on , vol.7, no.4, pp.329-334, October 1959.

[5] Dyson, J.;, "The characteristics and design of the conical log-spiral antenna," IEEE Trans Antenna Propag , vol.13, no.4, pp. 488- 499

[6] Jiwani, A.; Juswardy, B.; Padhi, S.; Bij de Vaate, J.G.; Hall, P.; , "Active antenna development for the SKA," Microwave Conference Proceedings (APMC), 2011 Asia-Pacific , vol., no., pp.1186-1189, 5-8 Dec. 2011

[7] Rumsey, V.; , "Frequency independent antennas," IRE International Convention Record , vol.5, no., pp. 114- 118, Mar 1957

[8] Jiwani, A.; Flexman, J.; Padhi, S.; de Vaate, J.G.B.; and Hall, P.J.; “An outdoor antenna metrology facility for candidate Square Kilometre Array antennas”, EMCSA 2011, Perth, 9 - 11 Nov. 2011 